\title{
Effects of Noisy Galvanic Vestibular Stimulation on Standing Balance - No Evidence for Stochastic Resonance in Young Healthy Adults
}

Lorenz Assländer ( $\sim$ lorenz.asslaender@uni-konstanz.de )

University of Konstanz

Louis-Solal Giboin

University of Konstanz

Markus Gruber

University of Konstanz

Roman Schniepp

Ludwig Maximilian University of Munich

Max Wühr

Ludwig Maximilian University of Munich

\section{Research Article}

Keywords: balance control, body sway, stochastic resonance, vestibular, galvanic vestibular stimulation

Posted Date: February 8th, 2021

DOI: https://doi.org/10.21203/rs.3.rs-154031/v1

License: (9) This work is licensed under a Creative Commons Attribution 4.0 International License.

Read Full License 


\section{Abstract}

Noisy galvanic vestibular stimulation (nGVS) at imperceptible levels has been shown to reduce body sway. This improvement was commonly attributed to the mechanism of stochastic resonance (SR). However, it has never been explicitly tested whether nGVS-induced effects on body sway consistently follow a SR-like bell-shaped performance curve with optimal improvements at intermediate noise intensities. To test this, body sway in 21 young healthy participants was measured during varying nGVS amplitudes while standing with eyes closed in 3 conditions (quiet stance, sway referencing, sinusoidal platform tilts). Presence of SR-compatible response dynamics in each trial was assessed (1) by a goodness-of-fit analysis using an established SR-curve model and (2) by ratings from 3 human experts. In accordance to theory, we found reductions of body sway at one nGVS amplitude in most trials (7595\%). However, only few trials exhibited SR-compatible bell-shaped performance curves with increasing noise amplitudes (10-33\%). Instead, body sway measures rather fluctuated randomly across nGVS amplitudes. This implies that, at least in young healthy adults, nGVS effects on body sway are incompatible with SR. Thus, previously reported reductions of body sway at particular nGVS intensities more likely result from inherent variations of the performance metric or by other yet unknown mechanisms.

\section{Introduction}

Stochastic resonance (SR) is a phenomenon in non-linear systems, where the system's response to weak, sub-threshold signals becomes enhanced in the presence of a weak stochastic interference, i.e., a particular non-zero amount of noise. ${ }^{1,2}$ Typically, SR effects will critically depend on the noise amplitude insofar as signal transfer becomes improved at intermediate noise intensities whereas too low or too high noise amplitudes will either not affect or disturb information processing within the system. Accordingly, the characteristic signature for the presence of SR is a noise-induced modulation of the system's output that follows a pseudo-bell-shaped performance curve, which peaks at some particular level of noise that optimally facilitates signal transfer within the system. ${ }^{3,4}$

SR-like effects have been demonstrated in animal models at the afferent receptor level for a variety of sensory systems, such as the somatosensory, auditory, and visual system. ${ }^{5-9}$ Similar effects have been later verified in humans in terms of improvements at the perceptual level as well as in related sensorimotor function. ${ }^{3,10-14}$ More recently, analogous effects have been observed in the vestibular system (for a review see ${ }^{15}$ ). To induce SR in the vestibular system, most studies used galvanic vestibular stimulation (GVS). GVS is a non-invasive technique that allows to electrically modulate the neuronal activity in the peripheral vestibular endorgans. ${ }^{16}$ By applying a noisy form of GVS (nGVS) to healthy young individuals, Galvan-Garza and colleagues could demonstrate that vestibular motion perception can get effectively enhanced in the presence of a low-intensity stochastic vestibular stimulation. ${ }^{17}$ Accordingly, they observed that in about $75 \%$ of participants nGVS-induced modulations of vestibular perceptual thresholds followed a bell-shaped performance curve with increasing noise amplitude. In 
agreement with the aforementioned theoretical framework of SR the authors observed a peak at an intermediate "optimal" nGVS intensity. These observations were later confirmed and extended in subsequent studies. ${ }^{18-20}$

To date, vestibular SR effects have been most extensively studied in the context of human balance control. Beneficial effects of nGVS on human postural control have been reported for young and older healthy individuals ${ }^{21,22}$ as well as in patients with peripheral vestibular hypofunction ${ }^{23}$ or central neurodegenerative diseases. ${ }^{24}$ These reports have been complemented by studies that specifically analyzed the effects of different stimulus characteristics, ${ }^{25,26}$ varying conditions of standing ${ }^{27-29}$ as well as immediate and after effects of stimulation ${ }^{30-33}$ on spontaneous sway patterns. In light of these studies, vestibular SR has been proposed to have immediate clinical implications in terms of a potential treatment option for postural imbalance in the context of a peripheral and/or central vestibular dysfunction. ${ }^{15}$

One common shortcoming of previous reports on nGVS effects on balance is, however, that they didn't provide sufficient evidence that the observed beneficial effects of nGVS on balance control are actually compatible with the presence of vestibular SR. Accordingly, neither of these studies demonstrated that nGVS-induced modulations of body sway consistently follow a bell-shaped performance curve with increasing nGVS amplitude. Rather, some studies examined the effects of only one fixed nGVS amplitude versus sham stimulation. ${ }^{22,26}$ Others studied the effects of different nGVS amplitudes but only reported performance modulations at the amplitude at which individuals showed optimally improved balance. ${ }^{23,31}$ This approach, however, is statistically problematic since a seeming improvement in performance across several repeated assessments would be likely expected simply due to inherent test-retest variations in the performance metric. Thus, both approaches fail to provide convincing evidence that reported improvements are linked to the mechanism of SR.

The aim of this study was therefore to examine whether previously reported nGVS-induced improvements in balance are consistent with and may be explained by the exhibition of SR. We hypothesized that, in analogy to previously reported effects of nGVS on vestibular perception, ${ }^{17}$ nGVS-induced reductions of body sway would follow a bell-shaped performance curve with increasing stimulation intensity consistent with the presence of SR. To this end, we systematically analyzed modulations of body sway in dependence of varying intensities of nGVS in young healthy individuals. nGVS-induced changes in body sway were studied in eyes closed stance during three surface conditions: fixed, sway referenced, and sinusoidally tilting. We applied different quantitative and qualitative criteria on a single subject level to determine whether nGVS-induced modulations in body sway were consistent with the presence of SR or rather follow other response dynamics.

\section{Materials And Methods}

\section{Participants}


Twenty-one healthy young subjects (age: $24.0 \pm 4.2$ years; height: $175 \pm 10 \mathrm{~cm}$; weight: $69.5 \pm 12.4 \mathrm{~kg}, 11$ females) participated in this study. None of the participants reported any auditory, vestibular, cardiovascular, or orthopedic disorders. All participants gave their written informed consent prior to the experiments. The Ethics Committee of the Ludwig-Maximilians-University approved the study protocol, which was conducted in conformity with the Declaration of Helsinki.

\section{Experimental setup}

During experiments, subjects stood either on two force plates (AMTI, Watertown, USA) or on a custombuilt device which allowed to control the tilt angle. Body sway was measured using a camera-based motion capture system (Vicon, Cambridge, UK). Reflective markers were attached to the sacrum, between the shoulder blades, and - not used for analyses - at head and shoulders (Fig. 1A). Stimuli were generated using Matlab scripts and a Simulink model (The Mathworks, Natick, USA) running on a realtime Target PC (Speedgoat, Switzerland). Analog (stimuli, force plates) and camera data were recorded on a PC running Nexus software (Vicon, Cambridge, UK) at $2000 \mathrm{~Hz}$ (down-sampled to $100 \mathrm{~Hz}$ for the analysis) and $100 \mathrm{~Hz}$ sampling rate, respectively. Rubber-electrodes (approx. $10 \mathrm{~cm}^{2}$ area) with electrode gel were applied to the mastoid process behind each ear and fixed with a head band to deliver bipolar, binaural GVS, which was generate by an isolated bipolar current stimulator (Digitimer, Hertfordshire, UK).

\section{Galvanic stimulus sequences and platform conditions}

Zero mean white noise sequences with a variance of $1 \mathrm{~mA}$ of 30-s or 60-s duration were generated using the 'wng' function (Matlab, 'Communications Toolbox') and filtered using a second order Butterworth band-pass filter with 0.02 and $10 \mathrm{~Hz}$ cutoff frequencies. The noise sequence was applied 9 consecutive times, with different scaling factors $(0 ; 0.1 ; 0.2 ; 0.3 ; 0.4 ; 0.5 ; 0.6 ; 1.0 ; 1.4)$, which resulted in signal rootmean-square (RMS) values of $0,14,28,43,57,71,85,142$, and $199 \mu \mathrm{A}$, which approximately correspond to peak-amplitude values of $0,0.05,0.10,0.15,0.20,0.25,0.30,0.50,0.70 \mathrm{~mA}$. All trials started with a 30 -s period without galvanic stimulation, followed by the $9 \mathrm{nGVS}$ amplitudes (including another 0-mA condition) in randomized order. The 60-s long trials (sine condition, see below) were split up into two trials with 4 and 5 different 60 -s long nGVS amplitudes. A new noise sequence and a new random order of stimulus amplitudes was generated for each subject.

The nine nGVS amplitudes were tested with eyes closed in three different platform conditions: fixed surface, sway referenced surface, and sinusoidal tilting surface (Fig. 1B). Sway referencing is a condition, in which the platform is commanded to follow the sway of the subject, such that orientation of the subjects' legs is not changing with respect to the platform. Sway referencing was achieved by measuring the movement of the hip via a sway-rod attached to a potentiometer, that was guided by a hook attached to the subjects' hip. Trigonometric calculations were used to calculate the command signal for the platform. As a result, proprioceptive information on body orientation with respect to the platform is decoupled from body orientation in space, making the information virtually useless for the balance task. The sine had a frequency of $0.5 \mathrm{~Hz}$ and $0.5^{\circ}$ amplitude and was started with a raised cosine to avoid platform jerks. Sway reference and sinusoidal tilt conditions were applied in separate trials for anterior- 
posterior (AP) and medio-lateral (ML) directions, while both directions were simultaneously recorded in quiet stance.

\section{Procedures}

After providing written informed consent, subjects' anthropometric measures were taken. Thereafter, we attached the reflective markers, hooks for sway referencing and nGVS electrodes after skin preparation with abrasive gel and disinfection. Subjects were asked to perform a 120-s long calibration routine in AP and $\mathrm{ML}$ direction (see below). Trials were presented in randomized order with short breaks of 1-2 minutes in between and longer breaks if requested by the subjects. Subjects were instructed to close the eyes and 'stand upright and comfortable' and were listening to non-rhythmic audio-books via noisecanceling head-phones to avoid auditory orientation cues and distract from the balancing task.

\section{Data analysis}

Center of pressure (COP) was calculated for quiet stance trials as output measure. Center of mass was calculated for sway referencing and sine trials as output measure, since the calculation of the COP was technically not feasible during moving platform conditions.

Center of mass (COM) was obtained from hip and shoulder movements using a calibration routine. ${ }^{34,35}$ During the calibration routine, subjects performed slow movements in the ankle and hip joints. Recorded COP trajectories were used as a projection of the COM position in these quasi-static trials. Assuming twosegment mechanics consisting of leg and head-arms-trunk segments, a regression between hip and ankle kinematics $\left(\mathrm{x}_{\mathrm{COP}} \approx \mathrm{x}_{\mathrm{COM}}=\mathrm{Off}+\mathrm{A} \cdot \mathrm{x}_{\mathrm{hip}}+\mathrm{B} \cdot \mathrm{x}_{\text {sho }}\right)$ provides calibration factors (Off, $\left.A, B\right)$, which can be used to obtain $x_{C O M}$ sway in conditions where $x_{C O M} \neq x_{C O P}$. Finally, COM angle is calculated from $x_{C O M}$ and COM height, as obtained from subject anthropometrics. ${ }^{36}$

To confirm the well documented effect of GVS evoking a postural response in ML direction, ${ }^{37}$ cumulant density functions between quiet stance COP and nGVS stimulus sequences with corresponding $95 \%$ confidence bounds were calculated for the first $28.16 \mathrm{~s}$ of each nGVS amplitude and across all subjects (using neurospec toolbox version $2.11^{38}$ ). For COP in quiet stance and COM in sway referenced platform conditions, path-length was calculated for each nGVS condition and subject using $p=1 / T \cdot \sum_{i}\left|x_{i+1}-x_{i}\right|$, where $T$ is the trial duration and $x_{i}$ are the individual samples. For the sine condition, body sway amplitude in response to the stimulus was obtained from the sway amplitude spectrum at the stimulus frequency calculated using a scaled Fast Fourier Transform. Data for individual subjects were normalized to body sway measures obtained during the $0-\mu \mathrm{A}$ nGVS condition and mean +/- standard deviation across subjects were calculated.

For each trial, we initially determined whether body sway measures improved for at least one particular nGVS level compared to baseline condition (i.e., $0 \mu \mathrm{A} n \mathrm{GVS}$ ). Since there is no established mathematical definition of what suffice for the exhibition of SR-like dynamics in response to varying levels of nGVS, we tested two alternative SR criteria, both on the results of single individuals as well as on the group 
outcomes. For the first criterion, we performed a goodness-of-fit analysis using an established SR-curve fit on the relationship between nGVS levels and normalized body sway measures as proposed in GalvanGarca et al. ${ }^{17}$. The SR function fits a bell-shaped curve on the data based on an equation that was initially developed to describe the general phenomenon of $\mathrm{SR}^{4}$ and is given by:

$$
A=B+A_{0} * \frac{l_{a}}{q_{2}} * \frac{1}{\sqrt{4 r+\Omega^{2}}}
$$

with $\quad r=\frac{1}{\sqrt{2} * \pi} * l_{a} * e^{-\frac{l_{a}^{2}}{2 * q_{2}}}$

$$
\text { and } \quad q_{2}=q+d q
$$

where $A_{0}, \Omega$, and $I_{a}$ determine the depth of the bell, $B$ the y-axis offset and dq the x-axis offset. All five parameters were fitted to experimental data using a global optimization approach using the function 'fmincon' and 'GlobalSearch' option from the Matlab 'Global Optimization Toolbox'. Subsequently, we tested for each trial whether the SR curve fit can better explain the experimental data compared to a simple linear fit. The comparison was performed using F-test statistics to account for the trade-of between improved residuals and additional free parameters in each fit with $F_{3,4}=9.1172$ for $p<.05$. The second criterion was more subjective and based the evaluation of three experts that rated experimental data for the presence or absence of SR based on visual inspection of individual response dynamics and their corresponding linear and SR curve fits. ${ }^{17}$ This criterion required that at least two of the expert judges identified the presence of SR-like dynamics in a trial.

\section{Data availability}

The datasets generated and analyzed during the current study are available from the corresponding author on reasonable request.

\section{Results}

During all examined stance conditions (quiet stance, sway-referencing, sinusoidal tilt), participants were able to maintain stable balance, except of four single trials (two due to circulation problems and two due to repeated loss of balance during sway referencing) that were excluded from further analysis. During quiet stance with eyes closed, subjects showed slight forward body lean and typical body sway amplitudes (exemplary COM body sway of one participant is presented in Fig. 1C). Body sway was 3-4 times larger in the sway referenced condition (not shown) as compared to quiet stance on a fixed surface. During the sinusoidal tilt condition, platform movement evoked sway responses that were visible in the time domain for most subjects. However, they were superimposed by considerable random sway.

In a first step of analysis, we examined whether nGVS above a certain intensity level (range of applied intensities: 0-199 $\mu \mathrm{A}$ signal RMS) would evoke a postural response in analogy to previously reported effects of GVS on ML postural sway. ${ }^{37}$ Visual inspection of corresponding nGVS and body sway traces 
did not exhibit obvious patterns of covariance (Fig. 1C). Cumulant density estimates (Fig. 2), however, revealed significant coupling between nGVS and body sway at the two highest applied nGVS intensities (i.e., 142 and $199 \mu$ A signal RMS). This effect was only observed for body sway in ML but not in AP direction. Cumulant density estimates further exhibited a characteristic biphasic pattern with short and medium latency responses at around 350 and 700 ms in agreement with previous reports. ${ }^{39,40}$ Hence, this analysis confirmed that the range of applied nGVS stimuli encompassed both suprathreshold nGVS intensities that have a direct modulatory effect on body sway as well as subthreshold nGVS intensities that could potentially induce alterations in body sway via vestibular SR.

In a second step of analysis, we examined whether response dynamics of body sway across the range of applied nGVS intensities might be actually compatible with the presence of vestibular-induced SR. SReffects on body sway would be indicated by a pseudo-bell-shaped response curve with an optimal reduction of body sway at some particular intermediate intensity of nGVS. For this purpose, sway parameters expressing a measure of overall body sway, i.e., sway path-length for quiet and swayreferenced stance conditions and sway amplitude in response to the tilt stimulus for sinusoidal tilt condition, were normalized with respect to the baseline condition (i.e., $0 \mu \mathrm{A} n \mathrm{GVS}$ ) and plotted across the range of nGVS amplitudes. Figure 3 to 5 depict group-average and representative individual body sway responses to nGVS and corresponding linear and SR curve fits for the three different examined stance conditions (individual plots for all recorded trials are provided as supplemental material).

In accordance with previous reports, we found reductions of body sway at individual nGVS amplitudes in the majority of subjects and platform conditions ( $95 \%$ and $86 \%$ for quiet stance in AP and ML direction, respectively; $84 \%$ and $85 \%$ for sway-referencing in $\mathrm{AP}$ and $\mathrm{ML}$ direction, respectively; $75 \%$ and $86 \%$ for sinusoidal tilt condition in AP and ML direction, respectively). In contrast, however, visual inspection of individual response dynamics by three human judges did only identify a minority of trials where body sway responses exhibited a SR-like pseudo-bell-shaped performance curve across applied nGVS intensities (33\% and $10 \%$ for quiet stance in AP and ML direction, respectively; $33 \%$ and $20 \%$ for swayreferencing stance in AP and $M L$ direction, respectively; $25 \%$ and $24 \%$ for sinusoidal tilt condition in AP and $\mathrm{ML}$ direction, respectively). This observation was further supported by the finding that none of the SR-curve fits (both on an individual as well as on a group-average level) yielded a significantly better explanatory power as compared to the linear fits. In other words, body sway across tested nGVS amplitudes did not show any systematic changes, but rather showed random fluctuations at the groupaverage level.

\section{Discussion}

Several previous studies reported that human postural sway can be reduced by the application of a particular intensity of nGVS ${ }^{21-33}$ - an effect that was concordantly attributed to the exhibition of SR within vestibular signal transfer. However, none of these studies so far provided systematic evidence for the latter assumption. In this study, we therefore systematically examined whether SR-like changes in body sway across nGVS amplitudes can be actually observed in young healthy individuals. The 
exhibition of SR in a particular system is typically indicated by a noise-induced modulation of the system's output that follows a pseudo-bell-shaped performance curve with increasing noise intensity, which peaks at a particular optimal noise amplitude where the system's performance becomes optimally enhanced. In accordance with previous reports, we found reduced body sway (for all three tested balance conditions and AP as well as ML directions) at particular nGVS intensities in almost all participants. However, across the entire range of applied nGVS intensities, individual and grouped body sway modulations exhibited more or less random fluctuations and, in most cases, did not exhibit consistent performance curves. This observation suggests that, at least in young healthy individuals, postural improvements at particular nGVS amplitudes are likely to result from inherent variations in the performance metric (i.e., static body sway) rather than being caused by vestibular SR.

Vestibular feedback cues play a minor role during quiet stance on a fixed surface, where postural adjustments predominantly rely on somatosensory and - to a lesser extent - visual cues. ${ }^{35,41}$ We therefore focused on stance conditions that pronounce the role of vestibular feedback by (a) withdrawal of visual cues (eyes closure) and (b) by manipulating the proprioceptive reference to the Earth vertical through sinusoidal surface tilts or sway referencing. Even under these conditions we did not observe convincing evidence that nGVS induces SR-like reductions in body sway. Our observations are certainly limited by the focus on a young and healthy cohort, in which peripheral vestibular processing presumably operates at a near-to-optimal level and might thus leave little to no potential for externally induced improvements. It is thus conceivable, that SR-like improvements in vestibular balance control might only be observable in the elderly or in patients with vestibular hypofunction, where age-related or pathological vestibular hair cell degeneration has been associated to a decline in peripheral vestibular signal processing. ${ }^{42,43}$

However, SR-like effects using nGVS have been reported also for young healthy individuals. Galvan-Garca and colleagues observed in about $75 \%$ of young participants, that nGVS-induced modulations of the vestibular threshold for passive motion perception were compatible with SR and followed a bell-shaped performance curve with optimal improvement at a particular intermediate nGVS intensity. ${ }^{17}$ Analogously, it was demonstrated that nGVS effectively lowers the threshold to induce vestibulospinal reflex responses in about $90 \%$ of young participants. ${ }^{44}$ Thus, the question arises why SR-like behavior can be found in perception and simple reflexes, but not in standing balance.

One reason could be differences between the processing of vestibular cues in ego motion, sensorimotor reflexes, and balance regulation. For instance, vestibulo-ocular reflex thresholds apparently differ from thresholds for vestibular motion perception, in particular in the low frequency range, ${ }^{45,46}$ and exhibit different response dynamics to vestibular stimulation or visual-vestibular conflict. ${ }^{47,48}$ Differences become even more apparent when comparing processing of vestibular cues at the perceptual and reflex level to that during balance regulation. The reasons for this lie both in the intrinsic multisensory nature and the complex feedback control mechanisms, which constitute postural control. Accordingly, vestibular input in postural control becomes considerably filtered, distorted, or even masked by the interaction with other sensory cues (proprioceptive, visual), the feedback dynamics of the postural control system, the 
body's low-pass characteristics, and intrinsic noise in neural processing and muscular activations. ${ }^{49}$ In line with this, current models of postural control, assume that vestibular cues only become involved in balance regulation after multisensory fusion (in particular with proprioceptive cues) at a late processing stage that is close to the behavioral output. ${ }^{50,51}$ Thus, comparisons between nGVS effects on different vestibular-related functions and output measures need to be considered carefully.

Another reason could be that psycho-physical estimates of vestibular perceptual thresholds, which were previously shown to be responsive to $\mathrm{nGVS},{ }^{17}$ are designed to be particularly robust against intraindividual performance variations and yield excellent test-to-retest reliability. ${ }^{52,53}$ In contrast, test-to-retest variations in standing balance are considerable. ${ }^{54,55}$ Thus, inherent trial-to-trial variations in the examined performance metric rather than nGVS-induced effects might govern the response dynamics of static body sway across the range of applied nGVS intensities. However, earlier studies reported nGVS-induced reductions in body sway measures in the order of $10-40 \% .{ }^{23,25}$ The trial-to-trial variability of sway measures from our current recordings had a standard deviation of $2 \%$ for quiet stance, $17 \%$ for swayreferencing trials, and $20 \%$ for sinusoidal platform tilt trials. Thus, at least the test-to-retest reliability during quiet stance would have been high enough to identify the previously reported $10-40 \%$ nGVSinduced improvements.

Finally, we observed that significant and consistent nGVS-induced body sway responses started to occur in ML direction for noise RMS intensities at and above $142 \mu \mathrm{A}$ (approximately corresponding to nGVS peak-to-peak amplitudes of 500 to $700 \mu \mathrm{A}$ ). In accordance in nonhuman primates, neuronal detection thresholds of primary vestibular afferents for GVS applied on the bilateral mastoid processes - a setup analogous to ours - were estimated to lie between 400 to $600 \mu \mathrm{A}$ peak-to-peak. ${ }^{56}$ This suggest that vestibular SR in young healthy individuals with intact peripheral vestibular information processing, should be triggered, if any, by nGVS at amplitudes below these estimated peripheral thresholds. This assumption corresponds to the observation made by Galvan-Garca et al. that optimal nGVS-induced reduction in vestibular perceptual thresholds occurred at nGVS peak-to-peak amplitudes at or below 500 $\mu A .{ }^{17}$ In contrast, previous reports on nGVS-induced improvements of postural sway at stimulation amplitudes way above these estimated detection thresholds (e.g. $1000 \mu \mathrm{A}^{21,26,33}$ ) are therefore unlikely to be attributable to vestibular SR. In these instances, reductions of body sway presumably rather result from a postural stiffening/stabilization response in the presence of an external induced vestibular disturbance as opposed to an effective facilitation of vestibular balance regulation.

\section{Declarations}

\section{Acknowledgment}

The work was supported by the German Federal Ministry for Education and Science (BMBF, IFB 01E01401) and the University Foundation of the Ludwig-Maximilans-Unversity Munich.

\section{Authors' contributions}


All of the authors have taken part in the preparation of this manuscript, have reviewed the results, and have approved the final version of this manuscript. L.A., L.G., S.G., and M.W. conceptualized and designed the study. L.A. and L.G. collected data. All authors were involved analyzing and interpretation of the data. L.A. and M.W. wrote the paper.

\section{Competing interests}

The corresponding author states on behalf of all authors that there are no conflicts of interest.

\section{References}

1. Collins, J., Chow, C. C. \& Imhoff, T. T. Stochastic resonance without tuning. Nature. 376, 236-238 (1995).

2. McDonnell, M. D. \& Ward, L. M. The benefits of noise in neural systems: bridging theory and experiment. Nat Rev Neurosci. 12, 415-426 https://doi.org/10.1038/nrn3061 (2011).

3. Moss, F., Ward, L. M. \& Sannita, W. G. Stochastic resonance and sensory information processing: a tutorial and review of application. Clin Neurophysiol. 115, 267-281 (2004).

4. Gammaitoni, L., Hänggi, P., Jung, P. \& Marchesoni, F. Stochastic resonance. Rev Mod Phys. 70, 223287 https://doi.org/10.1103/RevModPhys.70.223 (1998).

5. Collins, J. J., Imhoff, T. T. \& Grigg, P. Noise-enhanced information transmission in rat SA1 cutaneous mechanoreceptors via aperiodic stochastic resonance. J Neurophysiol. 76, 642-645 (1996).

6. Lindner, J. F., Bennett, M. \& Wiesenfeld, K. Stochastic resonance in the mechanoelectrical transduction of hair cells. Phys Rev E Stat Nonlin Soft Matter Phys. 72, 051911 https://doi.org/10.1103/PhysRevE.72.051911 (2005).

7. Jaramillo, F. \& Wiesenfeld, K. Mechanoelectrical transduction assisted by Brownian motion: a role for noise in the auditory system. Nat Neurosci. 1, 384-388 https://doi.org/10.1038/1597 (1998).

8. Funke, K., Kerscher, N. J. \& Worgotter, F. Noise-improved signal detection in cat primary visual cortex via a well-balanced stochastic resonance-like procedure. Eur J Neurosci. 26, 1322-1332 https://doi.org/10.1111/j.1460-9568.2007.05735.x (2007).

9. Nadrowski, B., Martin, P. \& Julicher, F. Active hair-bundle motility harnesses noise to operate near an optimum of mechanosensitivity. Proc Natl Acad Sci U S A. 101, 12195-12200 https://doi.org/10.1073/pnas.0403020101 (2004).

10. Richardson, K. A., Imhoff, T. T., Grigg, P. \& Collins, J. J. Using electrical noise to enhance the ability of humans to detect subthreshold mechanical cutaneous stimuli. Chaos. 8, 599-603 https://doi.org/10.1063/1.166341 (1998).

11. Zeng, F. G., Fu, Q. J. \& Morse, R. Human hearing enhanced by noise. Brain Res. 869, 251-255 (2000).

12. Simonotto, E. et al. Visual perception of stochastic resonance. Phys Rev Lett. 78, 1186 (1997).

13. Priplata, A. A., Niemi, J. B., Harry, J. D., Lipsitz, L. A. \& Collins, J. J. Vibrating insoles and balance control in elderly people. Lancet. 362, 1123-1124 https://doi.org/10.1016/S0140-6736(03)14470-4 
(2003).

14. Lipsitz, L. A. et al. A shoe insole delivering subsensory vibratory noise improves balance and gait in healthy elderly people. Arch Phys Med Rehabil. 96, 432-439 https://doi.org/10.1016/j.apmr.2014.10.004 (2015).

15. Wuehr, M., Decker, J. \& Schniepp, R. Noisy galvanic vestibular stimulation: an emerging treatment option for bilateral vestibulopathy. J Neurol. 264, 81-86 https://doi.org/10.1007/s00415-017-8481-4 (2017).

16. Dlugaiczyk, J., Wuehr, M. \& Straka, H. in Reference Module in Neuroscience and Biobehavioral Psychology(Elsevier, 2020).

17. Galvan-Garza, R. C., Clark, T. K., Mulavara, A. P. \& Oman, C. M. Exhibition of stochastic resonance in vestibular tilt motion perception. Brain Stimul. 11, 716-722 https://doi.org/10.1016/j.brs.2018.03.017 (2018).

18. Keywan, A., Wuehr, M., Pradhan, C. \& Jahn, K. Noisy Galvanic Stimulation Improves Roll-Tilt Vestibular Perception in Healthy Subjects. Front Neurol. 9, https://doi.org/10.3389/fneur.2018.00083 (2018).

19. Keywan, A., Jahn, K. \& Wuehr, M. Noisy Galvanic Vestibular Stimulation Primarily Affects OtolithMediated Motion Perception. Neuroscience. 399, 161-166 https://doi.org/10.1016/j.neuroscience.2018.12.031 (2019).

20. Keywan, A., Badarna, H., Jahn, K. \& Wuehr, M. No evidence for after-effects of noisy galvanic vestibular stimulation on motion perception. Sci Rep. 10, 2545 https://doi.org/10.1038/s41598-02059374-9 (2020).

21. Inukai, Y. et al. Effect of noisy galvanic vestibular stimulation on center of pressure sway of static standing posture. Brain Stimul. 11, 85-93 https://doi.org/10.1016/j.brs.2017.10.007 (2018).

22. Inukai, Y. et al. Effect of noisy galvanic vestibular stimulation in community-dwelling elderly people: a randomised controlled trial. J Neuroeng Rehabil. 15, 63-63 https://doi.org/10.1186/s12984-0180407-6 (2018).

23. Iwasaki, S. et al. Noisy vestibular stimulation improves body balance in bilateral vestibulopathy. Neurology. 82, 969-975 https://doi.org/10.1212/WNL.0000000000000215 (2014).

24. Samoudi, G., Jivegard, M., Mulavara, A. P. \& Bergquist, F. Effects of Stochastic Vestibular Galvanic Stimulation and LDOPA on Balance and Motor Symptoms in Patients With Parkinson's Disease. Brain Stimul. 8, 474-480 https://doi.org/10.1016/j.brs.2014.11.019 (2015).

25. Mulavara, A. P. et al. Improving balance function using vestibular stochastic resonance: optimizing stimulus characteristics. Exp Brain Res. 210, 303-312 https://doi.org/10.1007/s00221-011-2633-z (2011).

26. Nooristani, M., Maheu, M., Bacon, B. A. \& Champoux, F. The importance of nGVS current density for postural control enhancement. Brain Stimul. 12, 1592-1594 https://doi.org/10.1016/j.brs.2019.07.022 (2019). 
27. Fujimoto, C. et al. Noisy galvanic vestibular stimulation has a greater ameliorating effect on posture in unstable subjects: a feasibility study. Sci Rep. 9, 17189 https://doi.org/10.1038/s41598-01953834-7 (2019).

28. Piccolo, C., Bakkum, A. \& Marigold, D. S. Subthreshold stochastic vestibular stimulation affects balance-challenged standing and walking. PloS one. 15, e0231334 https://doi.org/10.1371/journal.pone.0231334 (2020).

29. Inukai, Y., Miyaguchi, S., Kobayashi, N., Otsuru, N. \& Onishi, H. Noisy galvanic vestibular stimulation effect on center of pressure sway during one-legged standing. J ClinNeurosci. 82, 173-178 https://doi.org/10.1016/j.jocn.2020.10.050 (2020).

30. Fujimoto, C. et al. Noisy Galvanic Vestibular Stimulation Sustainably Improves Posture in Bilateral Vestibulopathy. Front Neurol. 9, 900 https://doi.org/10.3389/fneur.2018.00900 (2018).

31. Fujimoto, C. et al. Noisy galvanic vestibular stimulation induces a sustained improvement in body balance in elderly adults. Sci Rep. 6, 37575 https://doi.org/10.1038/srep37575 (2016).

32. Inukai, Y. et al. The after-effect of noisy galvanic vestibular stimulation on postural control in young people: a randomized controlled trial. Neurosci Lett. 135009, https://doi.org/10.1016/j.neulet.2020.135009 (2020).

33. Nooristani, M., Maheu, M., Houde, M. S., Bacon, B. A. \& Champoux, F. Questioning the lasting effect of galvanic vestibular stimulation on postural control. PloS one. 14, e0224619-e0224619 https://doi.org/10.1371/journal.pone.0224619 (2019).

34. Assländer, L. \& Peterka, R. J. Sensory reweighting dynamics following removal and addition of visual and proprioceptive cues. J Neurophysiol. 116, 272-285 https://doi.org/10.1152/jn.01145.2015 (2016).

35. Peterka, R. J. Sensorimotor integration in human postural control. J Neurophysiol. 88, 1097-1118 (2002).

36. Winter, D. A. Biomechanics and motor control of human movement. 4th edn(Wiley, 2009).

37. Fitzpatrick, R. C. \& Day, B. L. Probing the human vestibular system with galvanic stimulation. J Appl Physiol (1985). 96, 2301-2316 https://doi.org/10.1152/japplphysiol.00008.2004 (2004).

38. Halliday, D. et al. A framework for the analysis of mixed time series/point process data-theory and application to the study of physiological tremor, single motor unit discharges and electromyograms. Prog Biophys Mol Biol. 64, 237-278 (1995).

39. Dakin, C. J., Luu, B. L., van den Doel, K., Inglis, J. T. \& Blouin, J. S. Frequency-specific modulation of vestibular-evoked sway responses in humans. J Neurophysiol. 103, 1048-1056 https://doi.org/10.1152/jn.00881.2009 (2010).

40. Dietrich, H. et al. Head motion predictability explains activity-dependent suppression of vestibular balance control. Sci Rep. 10, 668 https://doi.org/10.1038/s41598-019-57400-z (2020).

41. Fitzpatrick, R. \& McCloskey, D. I. Proprioceptive, visual and vestibular thresholds for the perception of sway during standing in humans. J Physiol. 478 (Pt 1), 173-186 https://doi.org/10.1113/jphysiol.1994.sp020240 (1994). 
42. Priesol, A. J., Valko, Y., Merfeld, D. M. \& Lewis, R. F. Motion Perception in Patients with Idiopathic Bilateral Vestibular Hypofunction. Otolaryngol Head Neck Surg. 150, 1040-1042 https://doi.org/10.1177/0194599814526557 (2014).

43. Bermúdez Rey, M. C. et al. Vestibular Perceptual Thresholds Increase above the Age of 40. Front Neurol. 7, 162-162 https://doi.org/10.3389/fneur.2016.00162 (2016).

44. Wuehr, M. et al. Stochastic resonance in the human vestibular system - Noise-induced facilitation of vestibulospinal reflexes. Brain Stimul. 11, 261-263 https://doi.org/10.1016/j.brs.2017.10.016 (2018).

45. Haburcakova, C., Lewis, R. F. \& Merfeld, D. M. Frequency dependence of vestibuloocular reflex thresholds. J Neurophysiol. 107, 973-983 https://doi.org/10.1152/jn.00451.2011 (2012).

46. Seemungal, B. M., Gunaratne, I. A., Fleming, I. O., Gresty, M. A. \& Bronstein, A. M. Perceptual and nystagmic thresholds of vestibular function in yaw. J Vestib Res. 14, 461-466 (2004).

47. Pettorossi, V. E. et al. Prolonged asymmetric vestibular stimulation induces opposite, long-term effects on self-motion perception and ocular responses. J Physiol. 591, 1907-1920 https://doi.org/10.1113/jphysiol.2012.241182 (2013).

48. Seemungal, B. M., Masaoutis, P., Green, D. A., Plant, G. T. \& Bronstein, A. M. Symptomatic Recovery in Miller Fisher Syndrome Parallels Vestibular-Perceptual and not Vestibular-Ocular Reflex Function. Front Neurol. 2, 2 https://doi.org/10.3389/fneur.2011.00002 (2011).

49. van der Kooij, H. \& Peterka, R. J. Non-linear stimulus-response behavior of the human stance control system is predicted by optimization of a system with sensory and motor noise. J Comput Neurosci. 30, 759-778 https://doi.org/10.1007/s10827-010-0291-y (2011).

50. Hettich, G., Asslander, L., Gollhofer, A. \& Mergner, T. Human hip-ankle coordination emerging from multisensory feedback control. Hum Mov Sci. 37, 123-146 https://doi.org/10.1016/j.humov.2014.07.004 (2014).

51. Assländer, L., Hettich, G. \& Mergner, T. Visual contribution to human standing balance during support surface tilts. Hum Mov Sci. 41, 147-164 https://doi.org/10.1016/j.humov.2015.02.010 (2015).

52. Lee, T. L., Shayman, C. S., Oh, Y., Peterka, R. J. \& Hullar, T. E. Reliability of Vestibular Perceptual Threshold Testing About the Yaw Axis. Ear Hear. https://doi.org/10.1097/aud.0000000000000859 (2020).

53. Merfeld, D. M. Signal detection theory and vestibular thresholds: I. Basic theory and practical considerations. Exp Brain Res. 210, 389-405 https://doi.org/10.1007/s00221-011-2557-7 (2011).

54. Carpenter, M. G., Frank, J. S., Winter, D. A. \& Peysar, G. W. Sampling duration effects on centre of pressure summary measures. Gait Posture. 13, 35-40 https://doi.org/10.1016/S09666362(00)00093-X (2001).

55. Nordahl, S. H., Aasen, T., Dyrkorn, B. M. \& Eidsvik, S. \& Molvaer, O. I. Static stabilometry and repeated testing in a normal population. Aviat. Space Environ. Med. 71, 889-893 (2000).

56. Kwan, A., Forbes, P. A., Mitchell, D. E., Blouin, J. S. \& Cullen, K. E. Neural substrates, dynamics and thresholds of galvanic vestibular stimulation in the behaving primate. Nat Commun 10 1904, 
Figures

A
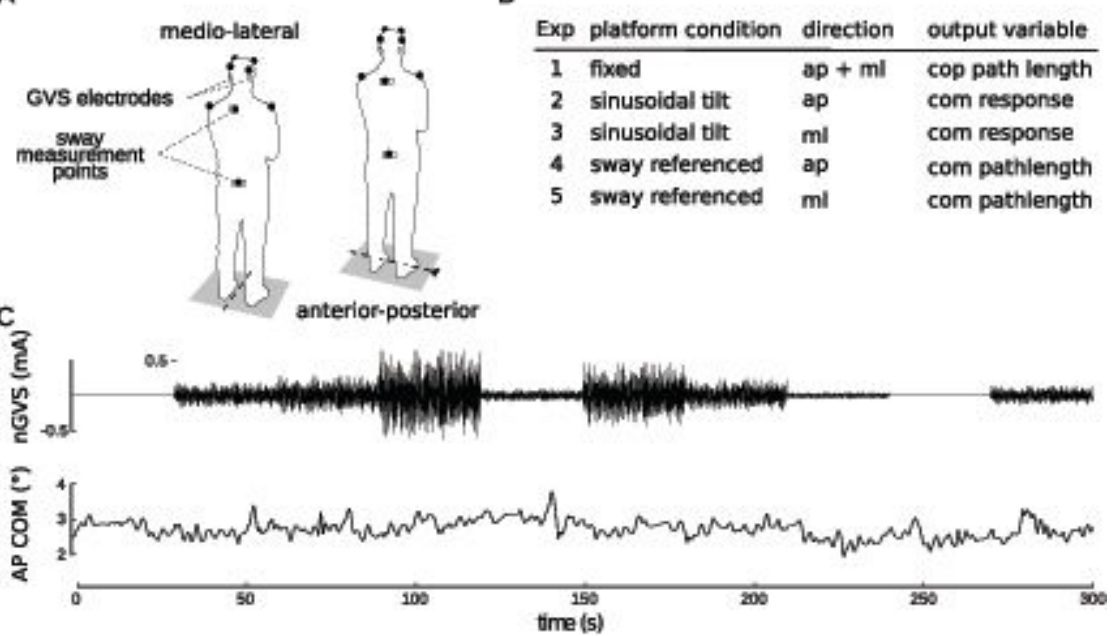

Figure 1

Summary of the experimental setup. A) the two stance positions on the tilt platform and marker placements. B) list of experimental conditions. C) example of the nGVS signal and anterior-posterior COM body sway during quiet stance. The order of nGVS amplitudes was randomized for each subject.
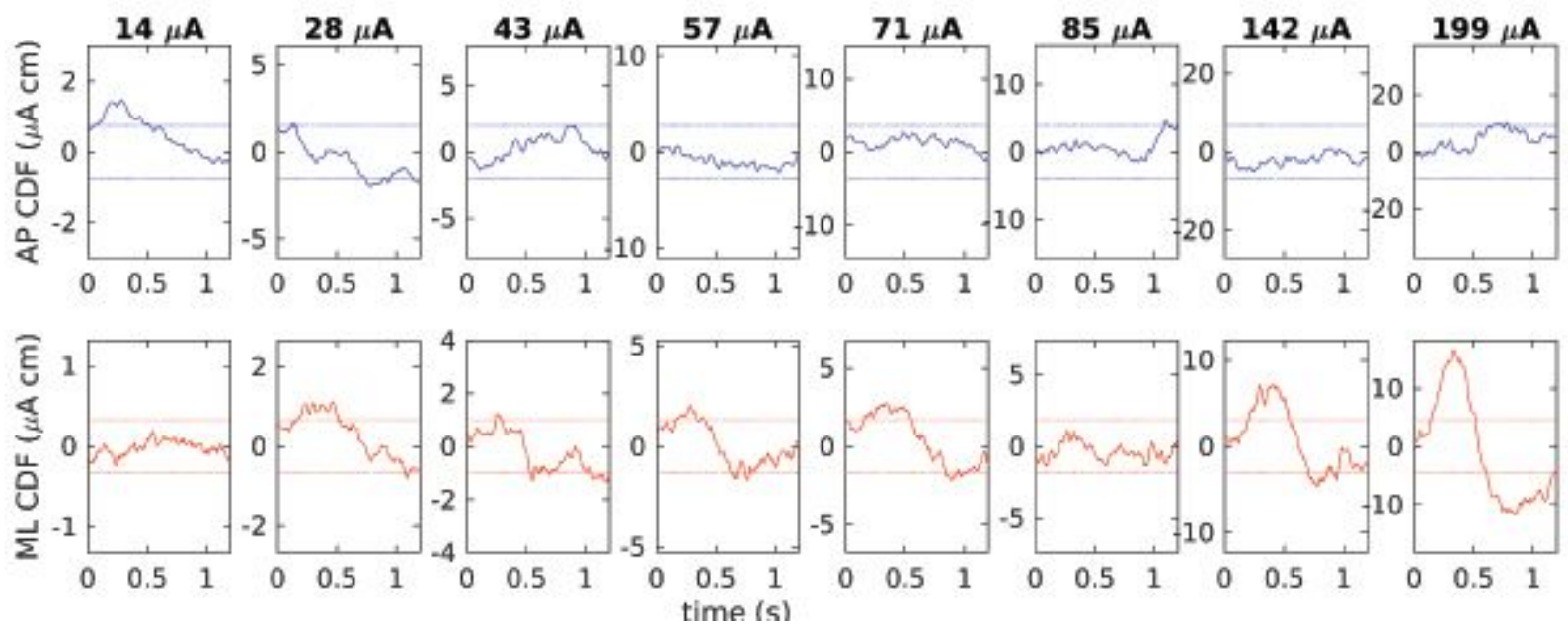

Figure 2

Cumulant density functions between COP and nGVS sequences for different stimulus amplitudes during quiet stance for anterior-posterior (blue, top row) and medio-lateral (red, bottom row) directions. Plots show the analysis results comprising all subjects. Dashed lines show 95\% confidence bounds; plots are scaled with respect to the confidence bounds. 

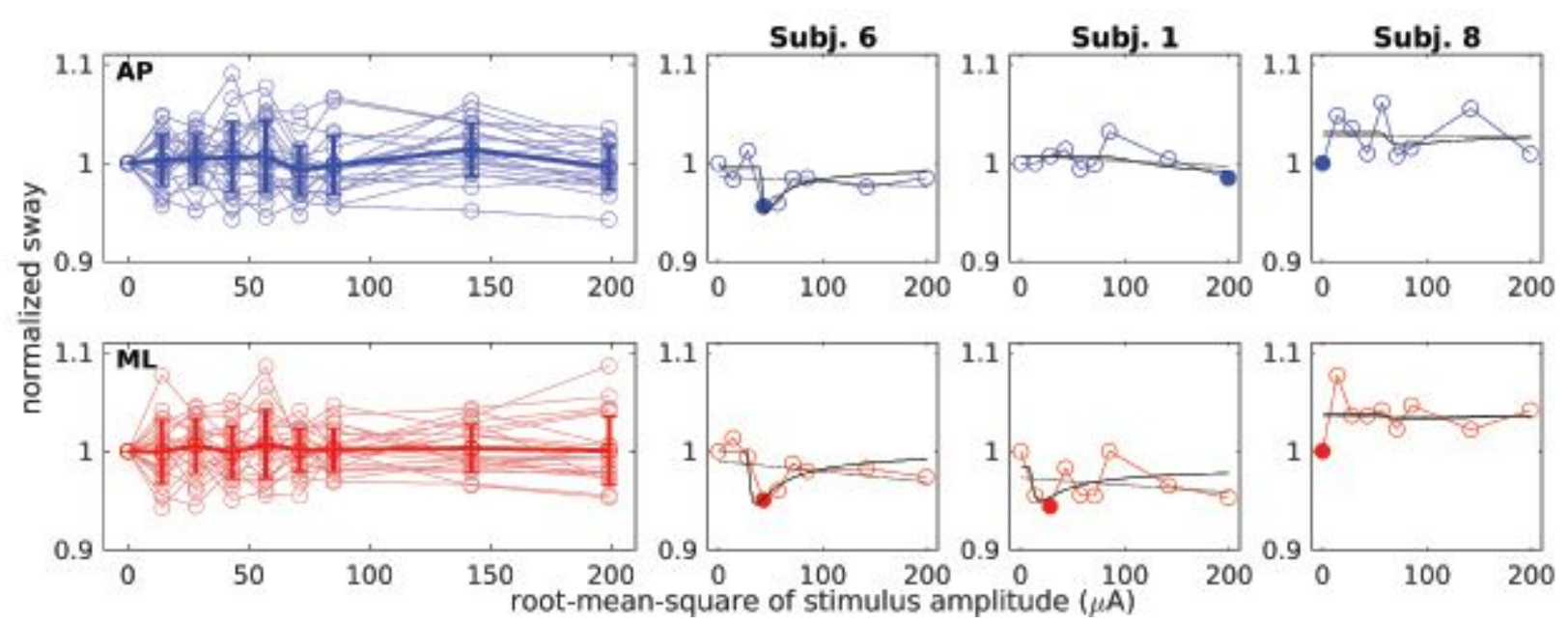

\section{Figure 3}

Normalized body sway path during quiet stance at varying nGVS intensities. Top row (AP, blue) shows anterior-posterior sway. Bottom row (ML, red) shows medio-lateral sway. (A) Responses of all subjects (light circles and lines) with group mean and standard deviation (thick line). (B-D) Three exemplary individual responses including the linear (grey) and the SR curve fit (black). Filled circles indicate minimum values. (B) Performance curve showing a SR-like bell-shaped with optimal improvement at an intermediate nGVS intensity. (C) Performance curve showing only optimal improvement at a particular nGVS intensity. (D) Performance curve showing none of the two.
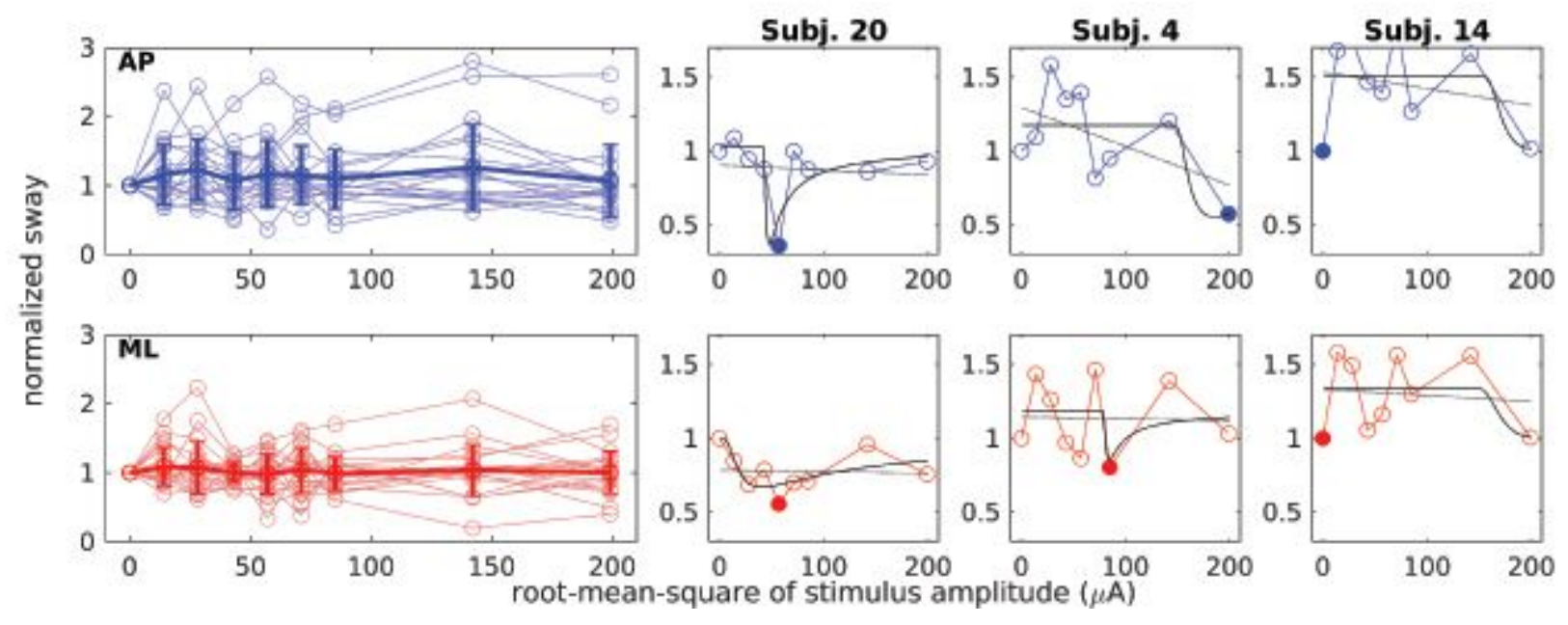

\section{Figure 4}

Normalized body sway path for standing with sway-referencing at varying nGVS intensities. Top row (AP, blue) shows anterior-posterior sway. Bottom row (ML, red) shows medio-lateral sway. (A) Responses of all subjects (light circles and lines) with group mean and standard deviation (thick line). (B-D) Three exemplary individual responses including the linear (grey) and the SR curve fit (black). Filled circles indicate minimum values. (B) Performance curve showing a SR-like bell-shaped with optimal improvement at an intermediate nGVS intensity. (C) Performance curve showing only optimal improvement at a particular nGVS intensity. (D) Performance curve showing none of the two. 

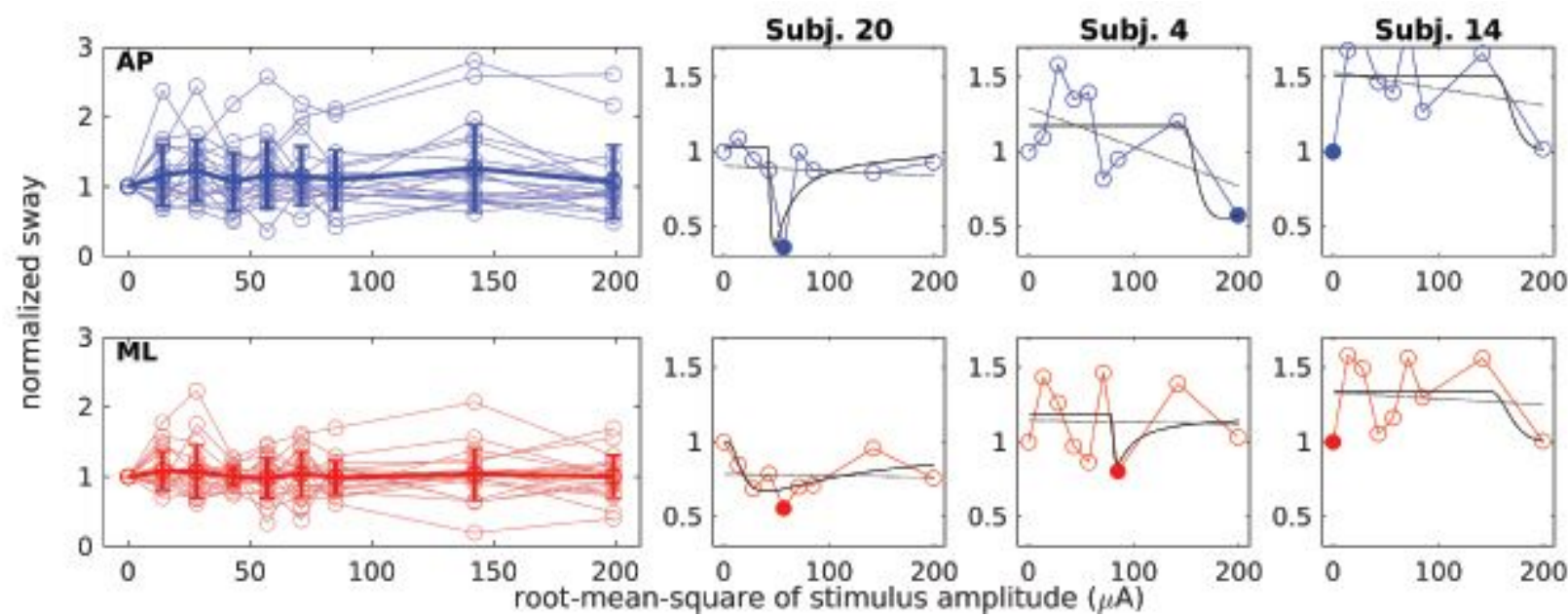

\section{Figure 5}

Normalized body sway path for standing during sinusoidal platform tilts at varying nGVS intensities. Top row (AP, blue) shows anterior-posterior sway. Bottom row (ML, red) shows medio-lateral sway. (A)

Responses of all subjects (light circles and lines) with group mean and standard deviation (thick line). (B-

D) Three exemplary individual responses including the linear (grey) and the SR curve fit (black). Filled circles indicate minimum values. (B) Performance curve showing a SR-like bell-shaped with optimal improvement at an intermediate nGVS intensity. (C) Performance curve showing only optimal improvement at a particular nGVS intensity. (D) Performance curve showing none of the two.

\section{Supplementary Files}

This is a list of supplementary files associated with this preprint. Click to download.

- Titlesupplementalfigures2.pdf

- singleSubjectFitsquietStance.pdf

- singleSubjectFitssine.pdf

- singleSubjectFitsswayRef.pdf 